\title{
A Flexible Software Defined Radio-based UHF RFID Reader Based on the USRP and LabView
}

\author{
Wang Yuechun, Ka Lok Man \\ Department of Computer Science \\ and Software Engineering \\ Xi'an Jiaotong-Liverpool University \\ Suzhou, China \\ yuechun.wang@xjtlu.edu.cn, \\ ka.man@xjtlu.edu.cn
}

\author{
Robert G. Maunder \\ School of Electronics and Computer \\ Science \\ University of Southampton \\ Southampton, United Kingdom \\ rm@ecs.soton.ac.uk
}

\author{
Jin Kyung Lee, Kyung Ki Kim \\ Department of Electronic \\ Engineering, \\ Daegu University, Gyeongsan, \\ South Korea \\ jklee@live.daegu.ac.kr, \\ kkkim@daegu.ac.kr
}

\begin{abstract}
This paper presents a UHF RFID Reader designed for recognition and tracking in IoT domain. It is built by NI USRP software radio platform and NI LabVIEW with flexible physical/MAC layer parameters, which can be modified easily and monitored clearly from front panel of this Reader compared to commercial RFID Reader. Queried random number sequence from a commercial Tag can be detected within half meter using this UHF Reader. All designs of this Reader are based on EPC Gen-2 RFID protocol, any further research based on this Reader can be easily connected and tested with commercial Tags.
\end{abstract}

Keywords; UHF RFID Reader, EPC Gen-2 RFID protocol, NI USRP, LabVIEW, Internet of Things

\section{INTRODUCTION}

Radio frequency identification (RFID) is one of the techniques that widely applied in recognition and item-level tracking tasks in Internet of Things domain [1]. UHF RFID supports significant wider read range, higher inventory rates, and rewritable product IDs [2]. Protocols used by RFID community in recent years are standardized for Electronic Product Code ${ }^{\mathrm{TM}}$ (EPC) to support the use of RFID [3]. Some researches aimed to provide complete control of physical and MAC layer based on these protocols have been done with NI USRP and Python-programmed GNU Radio [4,5], which will be challenge for some researchers. As a tool for teaching, LabVIEW is much easier with the advantages of using visual programming language.

This paper presents a UHF RFID Reader using USRP with LabVIEW software. An attempt to build flexible RFID Reader for less $\mathrm{C}++$ experienced researcher is presented in this paper. A UHF RFID Reader that can achieve several basic functions such as generating and sending commands, receiving and analyzing responses is presented. This Reader has flexible controlled parameters, which fits for studying principle of RFID Reader's operation on physical and MAC layer.

\section{FLEXIBLE RFID READER}

In this section, the theory and implementation of designed RFID Reader based on block diagram of Reader-Tag system (Figure 1) is presented. This system contains 6 parts and the Reader parts are core of this system.

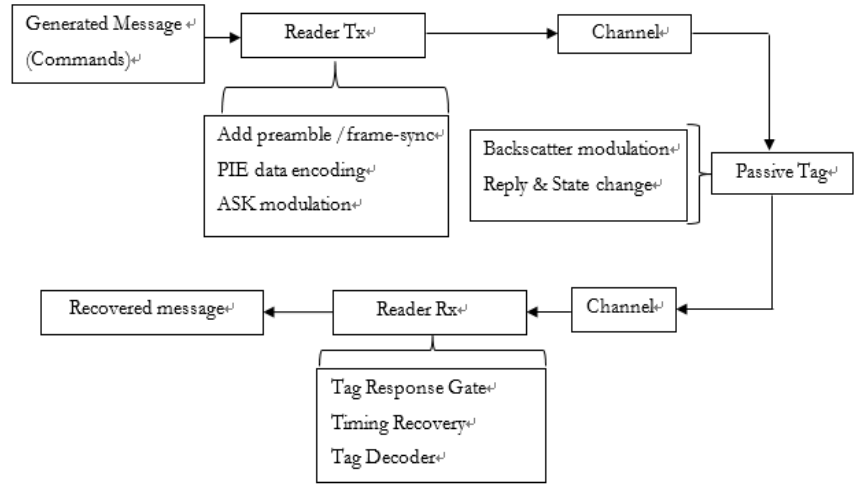

Figure 1. Block diagram of Reader-Tag system

\section{A. Overall Transceiver}

Structure of hardware part is built with two NI USRP, two RFID Circular antennas (PN6-868LCP/RCP), and one RFID passive tag (EURUHFT4928). Besides, Gigabit Ethernet cable is used to connect USRP with host PC. Compatible MIMO cable is used to link two USRPs and share the Ethernet. Antennas are PN6-868LCP/RCP which are chosen based on requirements of Gen-2 protocol. Avonwood EURUHFT4928 Tag is used for all experiments in this paper.

\section{B. Reader transmitter}

There are two functions supported by Reader transmitter: transmitting commands and providing energy to power up Tag.

As one significant character of passive Tag is that it acquires energy from transmitted signal of Reader, which denotes that Reader is required to transmit continuous waveform $(\mathrm{CW})$ between commands in order to keep Tag powered up. The simplest method is to keep USRP transmitting sequence of ones when the Transmit button is released. The size of transmitted continuous sequence is 100 bits in this paper. The choice of this value considered the system latency as well as the computing speed of LabVIEW. If this value is too high, it may cause the system latency because the receiver should receive all these ones before it stopped even when the expected signal has already been detected. If this value is too small, the USRP may stop working because the transmitter buffer becomes empty before the new command transmitted. 


\section{Reader receiver}

Block diagram of Reader Receiver chain is illustrated in Figure 2. Reader receiver catches both signals transmitted by Reader transmitter and backscattered by passive Tags.

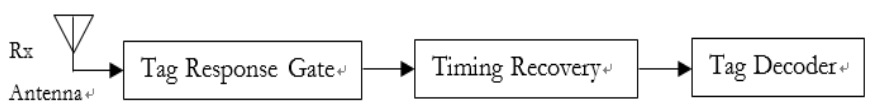

Figure 2. Block diagram of Reader Receiver

Tag Response Gate is used to detect and pass the useful information sequence to the downstream block. The detection technique is setting threshold in this paper. This block can reduce the computation of receiver because timing recovery and tag decoding only deal with expected information sequence rather than entire received signal which may include quantity of latency time. Synchronization of tag response is done in Timing Recovery block by calculating the cross-correlation of known preamble sequence and received tag response. Mission of Tag Decoder is to decode received RN16 sample sequence. Supposing backscatter from tag uses FM0 encoding format, key point of decoding FM0 encoded sequence is to identify the level reversal within one symbol. By observing the sequence of neighbor samples' difference within every data bit, original bit stream can be decoded. To match the transmitter and receiver, IQ rate and Carrier frequency in $\mathrm{Tx}$ parameters and $\mathrm{Rx}$ parameters should be the same. In this paper, IQ sampling rate is fixed as $400 \mathrm{kSps}$ and Carrier frequency is fixed as 867.6 MHz.

\section{OUTCOMES AND EVALUATION}

Parameter setting of $\mathrm{Tx}$ and $\mathrm{Rx}$ is listed in Table I, and entire signal sequence caught by receiver is illustrated in Figure 3, including Query and ACK command, Tag backscattered RN16, and the extended waveform of Query and ACK respectively, which shows no response to ACK command from Tag.

TABLE I. PARAMETERS SETTING OF TX AND RX

\begin{tabular}{|c|c|c|}
\hline Parameters & $\overline{T x}$ & $\mathbf{R x}$ \\
\hline Session Handle & 10.0.0.3 & $\overline{10.0 .0 .2}$ \\
\hline IQ sampling rate (Sps) & \multirow{2}{*}{\multicolumn{2}{|c|}{$\begin{array}{c}400 \mathrm{k} \\
867.6 \mathrm{M}\end{array}$}} \\
\hline Carrier Frequency $(\mathrm{Hz})$ & & \\
\hline Gain $(\mathrm{dB})$ & 25 & 0 \\
\hline Tari ( $\mu s)$ & 25 & - \\
\hline \# of samples & - & 300 \\
\hline
\end{tabular}

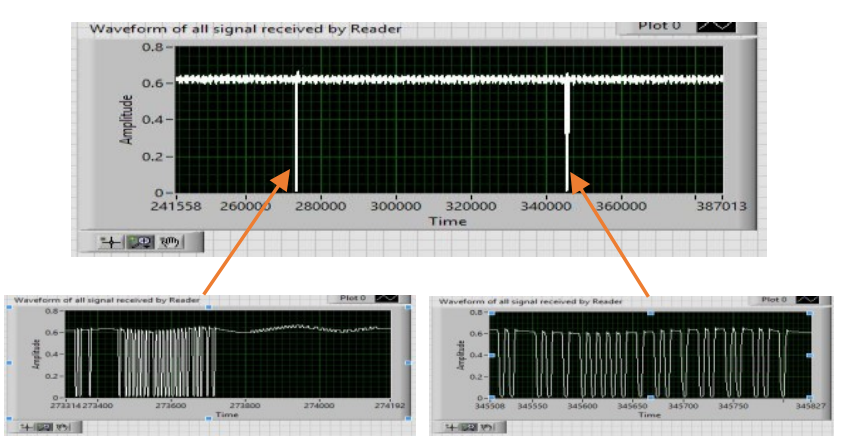

Figure 3. Entire signal sequence received by Reader
Reason of failed receiving Tag ID sequence is the delay between transmitted ACK command and RN16 sequence. Based on Gen-2 protocol, the maximum link timing after the last bit of RN16 and before the first bit of ACK command is 20TPri, where TPri denotes duration of one FM0 symbol or a single subcarrier cycle. In this case, delay between RN16 and ACK was over $500 \mu \mathrm{s} / 200$ samples, Tag will ignore ACK and no response will be backscattered.

To solve this problem, two attempts have been done. One attempt is to reduce the number of samples in each received frame. Another is to decline the size of array that transmitted to provide $\mathrm{CW}$ to Tag. Both are tried to decline that length of transmitted $\mathrm{CW}$ did not carry any information but just provide energy to Tag. However, when collecting the overall signal information using a feedback node in LabVIEW, both of these two attempts slowed done PC computing speed which finally resulted in LabVIEW stopped working because the transmit buffer became empty before the new data was provided. Besides, there are still several potential solving methods: one method is to use the 'Abort' function in LabVIEW which will stop receiver USRP keep receiving when expected information had been detected. This can allow transmitter sending ACK command as quick as possible after RN16 being detected rather than waiting all the $\mathrm{CW}$ sequences in transmit buffer have been received. Another method is to program LabVIEW in a lower level such as writing some $\mathrm{C}$ codes to allow the USRP sending $\mathrm{CW}$ rather than keep silence. If possible, using faster PC may solve this problem to a certain extent as well.

\section{CONCLUSION AND FUTURE WORK}

Implemented system achieved function of sending correct Reader-to-Tag commands under any adjustable parameter based on Gen-2 and decoding received Tag response. Due to computing speed of PC, response to ACK command from Tag has not finished till writing this paper. The potential research directions are studied and would be (1) to test actual environmental performance of Reader under variety communication channel by adding simulated noise to received signal in LabVIEW, (2) to build a passive Tag using USRP to simulate channel and two more USRPs may be used to act as transmitter and receiver of Tag.

\section{REFERENCES}

[1] Kubo, The Research of IoT Based on RFID Technology, in 2014 7th International Conference on Intelligent Computation Technology and Automation (ICICTA). 2014, 2014 7th IEEE International Conference: Changsha. p. 832-835

[2] Chiu, S., et al., A 900 MHz UHF RFID Reader Transceiver IC. SolidState Circuits, IEEE Journal of, 2007. 42(12): p. 2822-2833.

[3] Buettner, M. and D. Wetherall. A Flexible Software Radio Transceiver for UHF RFID Experimentation: UW TR: UW -CSE- 09- 10- 02.

[4] Buettner, M. and D. Wetherall. A software radio-based UHF RFID reader for PHY/MAC experimentation. in RFID (RFID), 2011 IEEE International Conference on. 2011.

[5] GS1, EPC Radio-Frequency Identity Protocols Generation-2 UHF RFID Specification for RFID Air Interface Protocol for Communication at 860 $\mathrm{MHz}$ - $960 \mathrm{MHz}$ Version 2.0.1 Ratified. 2015. 